\title{
Improved Algebraic Solution for Elliptic Localization in Distributed MIMO Radar
}

\author{
A. Noroozi ${ }^{1} \quad$ M. A. Sebt ${ }^{2} \quad$ A. H. Oveis ${ }^{3} \quad$ R. Amiri ${ }^{4} \quad$ M. M. Nayebi ${ }^{5}$ \\ ${ }^{1} \mathrm{PhD}$, Department of Electrical Engineering, Sharif University of Technology, Tehran, Iran. \\ ali_noroozi@sharif.edu \\ ${ }^{2}$ Associate Professor, Department of Electrical Engineering, K. N. Toosi University of Technology, Tehran, Iran. \\ sebt@kntu.ac.ir \\ ${ }^{3} \mathrm{PhD}$, Department of Electrical Engineering, K. N. Toosi University of Technology, Tehran, Iran. \\ oveis.amirhosein@ee.kntu.ac.ir \\ ${ }^{4} \mathrm{PhD}$, Department of Electrical Engineering, Sharif University of Technology, Tehran, Iran. \\ amiri_rouhollah@ee.sharif.edu \\ ${ }^{5}$ Professor,Department of Electrical Engineering, Sharif University of Technology, Tehran, Iran. \\ nayebi@sharif.edu
}

\begin{abstract}
:
In this paper, the problem of locating a target in a distributed multiple-input multiple-output radar system using bistatic range measurements is addressed. An algebraic closed-form two-stage weighted least squares solution for the considered problem is developed and analyzed. In the first stage, we establish a set of linear equations by eliminating the nuisance parameters first and then we apply a weighted least squares estimator to determine the target position estimate. In the second stage, in order to improve the localization performance and refine the solution of the first stage, an estimate of the target position estimation error is obtained. The final solution is obtained by subtracting the solution of the second stage from the solution of the first stage. The Cramer-Rao lower bound (CRLB) for target localization accuracy is developed in the case of Gaussian distribution. The proposed method is shown to be an approximately unbiased estimator, which is able to attain the CRLB accuracy under small noise conditions. Numerical simulations are included to examine the algorithm's performance and corroborate the theoretical developments.
\end{abstract}

Keywords: Bistatic Range (BR), Multiple-input Multiple-output (MIMO) Radar, Singular Value Decomposition (SVD), Target Position, Weighted Least Squares (WLS).

Submission date: 09, Sep. 2018

Conditional Acceptance date: 10, June. 2019

Acceptance date: 13, June. 2020

Corresponding author: Ali Noroozi

Corresponding author's address: Department of Electrical Engineering, Sharif University of Technology, Azadi

Tehran, Iran. 


\section{Introduction}

Multiple-input multiple-output (MIMO) radar systems with widely separated antennas have received significant attention over the past decade [1-6]. These radar systems referred to as distributed MIMO radars, take the advantage of the spatial diversity to improve detection performance and enhance target position estimation accuracy [7-15].

In recent years, several publications on target location estimation in the widely distributed MIMO radars have been presented, which indicate the importance of this issue. Generally, the localization methods in these radars are divided into two categories: direct and indirect methods. The latter methods first measure the time delays using the cross-ambiguity function. Then, by multiplying these time delays by the speed of signal propagation and performing some simple operations, the corresponding bistatic ranges (BRs), which is the sum of transmittertarget and target-receiver ranges, are calculated. These BRs form a set of elliptic equations through which the target position is obtained. In [16], a one-stage least squares (OSLS) solution is presented in which only a small number of the BR equations are employed and then these equations are converted to the RD ones. The target location is then obtained by the use of the resultant equations. Although the approach in [16] has a closedform solution, it does not utilize all the possible equations and cannot attain the Cramer-Rao lower bound (CRLB) accuracy. In [17], a closed-form two-step weighted least squares (TSWLS) method is developed to obtain the target position. The method can approximate the maximum likelihood estimator (MLE) under the conditions that the noise is small and the same in all the measurements. These conditions, however, are not reasonable from a practical viewpoint, especially the second one. In [18-20], two different closed-form onestage weighted least squares (OSWLS) methods for target localization are presented in the general case of noise in two different conditions - when the measurement noise is small, which leads to an approximate of the MLE, and when it is relatively high, which results in the best linear unbiased estimator. In [21], a closed-form one-step least squares (OSLS) method is presented, which is able to give the solution with fewer number of sensors (transmitters and receivers). Similar to the previous methods [16-20], the method in [21] is not able to reach the CRLB accuracy.

In this paper, we extend our previous work [20], in which a closed-form OSWLS solution is employed by eliminating the nuisance parameters through the singular value decomposition (SVD) approach, to a closed-form TSWLS solution. In this paper, in fact, we aim to refine the final solution in the second stage to improve the localization performance of the proposed method such that it can attain the CRLB accuracy.

The rest of this paper is organized as follows. Section 2 is devoted to the measurement model. The problem formulation from the $\mathrm{BR}$ measurements using $M$ transmitters and $N$ receivers is discussed in Section 3. The derivation of the proposed solution is also provided in this section. Section 4 first derives the error covariance matrix of the proposed method and the CRLB for the case of Gaussian distribution and then shows that the performance of the proposed method attains the CRLB. In Section 5, numerical simulations are included to evaluate performance of the proposed estimator. Finally, Section 6 concludes this paper with a summary.

The notations used throughout this paper are as follows. Vectors and matrices are represented by bold lower case and bold upper-case letters, respectively.

The superscripts ${ }^{T}$ and ${ }^{-1}$ stand for the transpose and inverse operators, respectively. $E\{\bullet\}$ and I $\|$ denotes the expectation operator and the Euclidean norm, respectively. $\operatorname{diag}(\mathbf{a})$ represents a diagonal matrix formed by the elements of $\mathbf{a}$. The notation $\operatorname{diag}\left(\mathbf{A}_{1}, \ldots, \mathbf{A}_{K}\right)$ stands for the block-diagonal matrix formed by $\mathbf{A}_{1}, \ldots, \mathbf{A}_{K}$ matrices. The $k$ th element of the vector $\mathbf{a}$ is represented by $\mathbf{a}(k)$, while the $k$ th row of the matrix $\mathbf{A}$ is denoted by $\mathbf{A}(k,:)$. The identity matrix of size $k \times k$ is denoted by $\mathbf{I}_{k}$. The noisy version or estimated value of the matrix $\mathbf{A}$ (or the vector $\mathbf{a}$ ) is $\hat{\mathbf{A}}$ (or $\hat{\mathbf{a}}$ ). In addition, the error term in the $\mathbf{A}$ (or $\mathbf{a}$ ) is represented by $\Delta \mathbf{A}$ (or $\Delta \mathbf{a})$.

\section{Measurement Model}

Consider a widely distributed MIMO radar system in which there is $M$ transmitters and $N$ receivers. The transmitters and receivers are located in a threedimensional (3-D) space, where the $i$ th transmitter is located at coordinates $\mathbf{x}_{t, i}=\left[x_{t, i}, y_{t, i}, z_{t, i}\right]^{T}, \quad i=1, \ldots, M$, and the $j$ th receiver is also located at coordinates $\mathbf{x}_{r, j}=\left[x_{r, j}, y_{r, j}, z_{r, j}\right]^{T}, j=1, \ldots, N$. We aim to determine the target position, $\mathbf{x}_{0}=\left[x_{0}, y_{0}, z_{0}\right]^{T}$. For this purpose, the transmitters send out a set of narrowband orthogonal waveforms. The transmitted signals are then reflected by the target of interest. In addition, the receivers collect the direct and the reflected signals from the transmitters and the target, respectively. It is important to note that we use the terms TD and BR interchangeably throughout this paper since there exists a unique linear relationship between them.

Let $\mathbf{r}$ be all the noise-free BR measurements expressed as

$\mathbf{r}=\hat{\mathbf{r}}-\Delta \mathbf{r}$

where $\quad \mathbf{r}=\left[\begin{array}{lll}\mathbf{r}_{1}^{T} & \ldots & \mathbf{r}_{M}^{T}\end{array}\right]^{T} \quad$ and $\quad \mathbf{r}_{i}=\left[\begin{array}{lll}r_{i, 1} & \ldots & r_{i, N}\end{array}\right]^{T}$ denotes the noise-free BR measurements of the $i$ th transmitter, is the BR noise vector, $\hat{\mathbf{r}}=\left[\begin{array}{lll}\hat{\mathbf{r}}_{1}^{T} & \ldots & \hat{\mathbf{r}}_{M}^{T}\end{array}\right]^{T}$ and $\quad \hat{\mathbf{r}}_{i}=\left[\begin{array}{lll}\hat{r}_{i, 1} & \ldots & \hat{r}_{i, N}\end{array}\right]^{T} \quad$ represents the $\mathrm{BR}$ 
measurements of the $i$ th transmitter, is the BR noise vector, and $\Delta \mathbf{r}=\left[\begin{array}{lll}\Delta \mathbf{r}_{1}^{T} & \ldots & \Delta \mathbf{r}_{M}^{T}\end{array}\right]^{T}$ denotes the noise which is present in the BR measurements of the $i$ th transmitter. The BR noise vector $\Delta \mathbf{r}$ is assumed to be Gaussian with zero mean and covariance matrix

$$
\mathbf{Q}=E\left[\Delta \mathbf{r} \Delta \mathbf{r}^{T}\right]
$$

In the following section, first the localization problem is formulated based on the BR measurements and then a novel closed-form TSWLS solution is employed to find the target position.

\section{Closed-Form Solution}

Stage-1: The BR for the pair of $i$ th transmitter and the $j$ th receiver, which can be obtained by multiplying the corresponding measured time delay by the speed of signal propagation, is given by

$r_{i, j}=R_{t, i}+R_{r, j}$,

where $R_{t, i}=\left\|\mathbf{x}_{0}-\mathbf{x}_{t, i}\right\|$ and $R_{r, j}=\left\|\mathbf{x}_{0}-\mathbf{x}_{r, j}\right\|$ denote the ranges from the target to the $i$ th transmitter and the $j$ th receiver, respectively. By rearranging (3) as $r_{i, j}-R_{t, i}=R_{r, j}$, squaring both sides of the resultant expression and performing some algebraic manipulations, it follows that

$$
\left(\mathbf{x}_{t, i}^{T}-\mathbf{x}_{r, j}^{T}\right) \mathbf{x}_{0}=\frac{1}{2}\left(r_{i, j}^{2}+\mathbf{x}_{t, i}^{T} \mathbf{x}_{t, i}-\mathbf{x}_{r, j}^{T} \mathbf{x}_{r, j}\right)-r_{i, j} R_{t, i} . \text { (4) }
$$

For the set of $N$ receivers, (4) can be cast into matrix form as

$$
\mathbf{S}_{i} \mathbf{x}_{0}=\mathbf{z}_{i}+\mathbf{r}_{i} R_{t, i},
$$

where

$$
\mathbf{S}_{i}(j,:)=\mathbf{x}_{t, i}^{T}-\mathbf{x}_{r, j}^{T}, \mathbf{z}_{i}(j)=\frac{1}{2}\left(r_{i, j}^{2}+\mathbf{x}_{t, i}^{T} \mathbf{x}_{t, i}-\mathbf{x}_{r, j}^{T} \mathbf{x}_{r, j}\right) \text {. (6) }
$$

In (5), the target position $\mathbf{x}_{0}$ and the nuisance parameter $R_{t, i}$ are unknown. In fact, (5) represents a linear system with respect to $\mathbf{x}_{0}$ and $R_{t, i}$. Now, the nuisance parameter $R_{t, i}$ can be removed by premultiplying (5) by the matrix $\mathbf{M}_{i}$, of which the vector $\mathbf{r}_{i}$ is in the null space, which is given by [20]

$$
\mathbf{M}_{i}=\mathbf{V}^{T} \mathbf{D}_{i},
$$

where

$$
\mathbf{D}_{i}=\left(\operatorname{diag}\left(\mathbf{r}_{i}\right)\right)^{-1} \text {, }
$$

and the matrix $\mathbf{V}$ is obtained from the SVD of the matrix $\left(\mathbf{I}_{N}-\mathbf{Z}\right)$ given by [20]
$\left(\mathbf{I}_{N}-\mathbf{Z}\right)=\left[\begin{array}{ll}\mathbf{U} & \mathbf{u}\end{array}\right]\left[\begin{array}{cc}\boldsymbol{\Sigma}_{z} & \mathbf{0} \\ \mathbf{0}^{T} & 0\end{array}\right]\left[\begin{array}{c}\mathbf{V}^{T} \\ \mathbf{v}^{T}\end{array}\right]=\mathbf{U} \boldsymbol{\Sigma}_{z} \mathbf{V}^{T}$

$\mathbf{Z}=\left[\begin{array}{cc}\mathbf{0} & \mathbf{I}_{N-1} \\ 1 & \mathbf{0}^{T}\end{array}\right]_{N \times N}=$ circularshift matrix

where $\mathbf{U}$ and $\mathbf{V}$ are orthogonal matrices of length $N \times(N-1)$ corresponding to nonzero singular values of $\left(\mathbf{I}_{N}-\mathbf{Z}\right), \mathbf{u}$ and $\mathbf{v}$ are vectors spanning the null space of $\left(\mathbf{I}_{N}-\mathbf{Z}\right)$ and $\left(\mathbf{I}_{N}-\mathbf{Z}\right)^{T}, \quad$ respectively, and corresponding to zero singular value of $\left(\mathbf{I}_{N}-\mathbf{Z}\right)$, and $\boldsymbol{\Sigma}_{z}$ is a diagonal matrix with $N-1$ nonzero singular values of $\left(\mathbf{I}_{N}-\mathbf{Z}\right)^{T}$. As $\left(\mathbf{I}_{N}-\mathbf{Z}\right) \mathbf{D}_{i} \mathbf{r}_{i}=\mathbf{0}$, it follows that $\mathbf{V}^{T} \mathbf{D}_{i} \mathbf{r}_{i}$ is zero as well. Thus, we have

$\mathbf{M}_{i} \mathbf{S}_{i} \mathbf{x}_{0}=\mathbf{M}_{i} \mathbf{z}_{i}$

Now, we extend the problem to the general case when there exist $M$ transmitters. After performing the above procedure for each of the transmitters and then stacking all the resultant expressions into matrix form, the problem can be represented as

$\mathbf{A}_{1} \mathbf{x}_{0}=\mathbf{b}_{1}$

where

$$
\begin{aligned}
& \mathbf{A}_{1}=\left[\begin{array}{lll}
\left(\mathbf{M}_{1} \mathbf{S}_{1}\right)^{T} & \ldots & \left(\mathbf{M}_{M} \mathbf{S}_{M}\right)^{T}
\end{array}\right]^{T}, \\
& \mathbf{b}_{1}=\left[\begin{array}{lll}
\left(\mathbf{M}_{1} \mathbf{z}_{1}\right)^{T} & \ldots & \left(\mathbf{M}_{M} \mathbf{z}_{M}\right)^{T}
\end{array}\right]^{T} .
\end{aligned}
$$

Since measurement errors are always present, we must take the noise into account in the measured values. By substituting $\mathbf{r}=\hat{\mathbf{r}}-\Delta \mathbf{r}$ into (12) and eliminating the second-order noise terms, we have

$\boldsymbol{\varepsilon}_{1}=\hat{\mathbf{b}}_{1}-\hat{\mathbf{A}}_{1} \mathbf{x}_{0}$,

where the error vector is given as follows

$$
\begin{aligned}
& \boldsymbol{\varepsilon}_{1}=\left[\begin{array}{lll}
\boldsymbol{\varepsilon}_{1,1}^{T} & \ldots & \boldsymbol{\varepsilon}_{1, M}^{T}
\end{array}\right]^{T}, \\
& \boldsymbol{\varepsilon}_{1, i} \approx \mathbf{V}^{T}\left(\mathbf{I}_{N}-\mathbf{D}_{i} R_{t, i}\right) \Delta \mathbf{r}_{i} .
\end{aligned}
$$

It is worth noting that (15) represents a linear relationship with respect to $\mathbf{x}_{0}$. Therefore, the unknown vector can be estimated by the WLS method. The WLS solution, which minimizes the cost function $\boldsymbol{\varepsilon}_{1}^{T} \mathbf{W}_{1} \boldsymbol{\varepsilon}_{1}$ to $\mathbf{x}_{0}$, is given by [22]

$$
\hat{\mathbf{x}}_{0}^{(1)}=\left(\hat{\mathbf{A}}_{1}^{T} \mathbf{W}_{1} \hat{\mathbf{A}}_{1}\right)^{-1} \hat{\mathbf{A}}_{1}^{T} \mathbf{W}_{1} \hat{\mathbf{b}}_{1}
$$

where $\hat{\mathbf{x}}_{0}^{(1)}$ denotes the target position estimate in the first stage and $\mathbf{W}_{1}$ is a symmetric positive definite matrix chosen here as 
$\mathbf{W}_{1}=E\left[\boldsymbol{\varepsilon}_{1} \boldsymbol{\varepsilon}_{1}^{T}\right]^{-1}=\left(\mathbf{B}_{1} \mathbf{Q} \mathbf{B}_{1}^{T}\right)^{-1}$,

where

$\mathbf{B}_{1}=\operatorname{blkdiag}\left(\mathbf{B}_{1,1}, \ldots, \mathbf{B}_{1, M}\right)$,

$\mathbf{B}_{1, i}=\mathbf{V}^{T}\left(\mathbf{I}_{N}-\mathbf{D}_{i} R_{t, i}\right)$ and $\mathbf{Q}$ is given by (2).

Stage-2: In order to refine the target position estimate obtained in the first stage, we aim to determine an estimate of the target position estimation error $\Delta \mathbf{x}_{0}^{(1)}$ and then subtract it from the solution of the first stage $\hat{\mathbf{x}}_{0}^{(1)}$. This can produce a substantial improvement in the performance of the proposed method. To this end, we substitute the terms $\mathbf{x}_{0}=\hat{\mathbf{x}}_{0}^{(1)}-\Delta \hat{\mathbf{x}}_{0}^{(1)}$ and $r_{i, j}=\hat{r}_{i, j}-\Delta r_{i, j}$ into (4) and expand $R_{t, i} \# \mathbf{x}_{0}-\mathbf{x}_{t, i} \|$ in a Taylor series and retain only the linear terms which is given by

$R_{t, i}=\left\|\mathbf{x}_{0}^{(1)}-\mathbf{x}_{t, i}-\Delta \hat{\mathbf{x}}_{0}^{(1)}\right\| \approx\left\|\mathbf{x}_{0}^{(1)}-\mathbf{x}_{t, i}\right\|-\boldsymbol{\rho}_{\mathbf{x}_{0}^{(1)}, \mathbf{x}_{t, i}}^{T} \Delta \hat{\mathbf{x}}_{0}^{(1)}$

where $\boldsymbol{\rho}_{\mathbf{a}, \mathbf{b}}:=(\mathbf{a}-\mathbf{b}) /\|\mathbf{a}-\mathbf{b}\|$ denotes the unit vector directed from $\mathbf{b}$ to $\mathbf{a}$. Therefore, after substituting (20) into (4) and ignoring the second- and higher-order error terms, (4) can be expressed as

$$
\begin{aligned}
\left(\hat{r}_{i, j}-\left\|\mathbf{x}_{0}^{(1)}-\mathbf{x}_{t, i}\right\|\right) \Delta r_{i, j} \approx & \frac{1}{2}\left(\mathbf{x}_{t, i}^{T} \mathbf{x}_{t, i}-\mathbf{x}_{r, j}^{T} \mathbf{x}_{r, j}+\hat{r}_{i, j}^{2}\right) \\
- & \left(\mathbf{x}_{t, i}-\mathbf{x}_{r, j}\right)^{T} \mathbf{x}_{0}^{(1)}-\hat{r}_{i, j}\left\|\mathbf{x}_{0}^{(1)}-\mathbf{x}_{t, i}\right\| \\
& +\left(\mathbf{x}_{t, i}-\mathbf{x}_{r, j}+\hat{r}_{i, j} \mathbf{\rho}_{\mathbf{x}_{0}^{(1)}, \mathbf{x}_{\mathrm{t}, i}}\right)^{T} \Delta \mathbf{x}_{0}^{(1)} .
\end{aligned}
$$

Doing (21) for all transmitters and receivers yields, in matrix form,

$\boldsymbol{\varepsilon}_{2} \approx \hat{\mathbf{b}}_{2}-\hat{\mathbf{A}}_{2} \Delta \mathbf{x}_{0}^{(1)}$,

where $\boldsymbol{\varepsilon}_{2}=\mathbf{B}_{2} \Delta \mathbf{r}$ and

$$
\hat{\mathbf{b}}_{2}(k)=\frac{1}{2}\left(\mathbf{x}_{t, i}^{T} \mathbf{x}_{t, i}-\mathbf{x}_{r, j}^{T} \mathbf{x}_{r, j}+\hat{r}_{i, j}^{2}\right)
$$$$
-\left(\mathbf{x}_{t, i}-\mathbf{x}_{r, j}\right)^{T} \hat{\mathbf{x}}_{0}^{(1)}-\hat{r}_{i, j}\left\|\hat{\mathbf{x}}_{0}^{(1)}-\mathbf{x}_{t, i}\right\|,
$$$$
\hat{\mathbf{A}}_{2}(k,:)=-\left(\mathbf{x}_{t, i}-\mathbf{x}_{r, j}+\hat{r}_{i, j} \mathbf{\rho}_{\hat{\mathbf{x}}_{0}^{(1)}, \mathbf{x}_{\mathrm{t}, \mathrm{i}}}\right)^{T} \text {, }
$$$$
\mathbf{B}_{2}(k, k)=\hat{r}_{i, j}-\left\|\hat{\mathbf{x}}_{0}^{(1)}-\mathbf{x}_{t, i}\right\|
$$

and $k=(i-1) N+j$. The WLS solution of (22) is obtained via

$$
\Delta \hat{\mathbf{x}}_{0}^{(1)}=\left(\hat{\mathbf{A}}_{2}^{T} \mathbf{W}_{2} \hat{\mathbf{A}}_{2}\right)^{-1} \hat{\mathbf{A}}_{2}^{T} \mathbf{W}_{2} \hat{\mathbf{b}}_{2},
$$

where $\mathbf{W}_{2}$ is the weighting matrix which is given by

$$
\mathbf{W}_{2}=E\left\{\boldsymbol{\varepsilon}_{2} \boldsymbol{\varepsilon}_{2}^{T}\right\}^{-1}=\left(\mathbf{B}_{2} \mathbf{Q} \mathbf{B}_{2}^{T}\right)^{-1} \text {. }
$$

Finally, the refined solution of the target position estimate in the second stage is obtained via

$\hat{\mathbf{x}}_{0}^{(2)}=\hat{\mathbf{x}}_{0}^{(1)}-\Delta \hat{\mathbf{x}}_{0}^{(1)}$.
The final point to note in this section is that $\mathbf{W}_{1}$ in (19) depends on the unknown nuisance parameters $R_{t, i}$, $i=1, \ldots, M$, through $\mathbf{B}_{1}$. To implement the proposed algorithm, we first set the weighting matrix as $\left(\mathbf{B}_{1}^{\prime} \mathbf{Q} \mathbf{B}_{1}^{\prime T}\right)^{-1}$, where $\mathbf{B}_{1}^{\prime}=\operatorname{blkdiag}\left(\mathbf{B}_{1,1}^{\prime}, \ldots, \mathbf{B}_{1, M}^{\prime}\right) \quad$ and $\mathbf{B}_{1, i}^{\prime}=\mathbf{V}^{T}$ for $i=1, \ldots, M$, to obtain an initial estimate of the nuisance parameters. Then, we employ the initial estimated value of the nuisance parameters $R_{t, i}$, $i=1, \ldots, M$, to compute the weighting matrix $\mathbf{W}_{1}$ according to (19).

\section{Performance Analysis}

In this section, we first derive the covariance matrix of the proposed method as well as the CRLB and then draw a comparison between them to check whether the proposed estimator can reach the CRLB accuracy or not.

\subsection{Error Covariance Matrix}

The error covariance matrix of the proposed estimator is $\operatorname{cov}\left(\hat{\mathbf{x}}_{0}^{(2)}\right)=E\left\{\left(\hat{\mathbf{x}}_{0}^{(2)}-E\left\{\hat{\mathbf{x}}_{0}^{(2)}\right\}\right)\left(\hat{\mathbf{x}}_{0}^{(2)}-E\left\{\hat{\mathbf{x}}_{0}^{(2)}\right\}\right)^{T}\right\}$

To determine (29), we express the estimated values in the first and second stages of the algorithm as $\hat{\mathbf{x}}_{0}^{(1)}=\mathbf{x}_{0}+\Delta \mathbf{x}_{0}^{(1)}$ and $\Delta \hat{\mathbf{x}}_{0}^{(1)}=\Delta \mathbf{x}_{0}^{(1)}+\delta \mathbf{x}_{0}^{(1)}$, respectively. Thus, we have

$\mathbf{x}_{0}^{(2)}-E\left\{\mathbf{x}_{0}^{(2)}\right\}=E\left\{\delta \mathbf{x}_{0}^{(1)}\right\}-\delta \mathbf{x}_{0}^{(1)}$.

Subtracting the actual value $\Delta \mathbf{x}_{0}^{(1)}$ from (26), performing some elementary manipulations and using (22), yields

$$
\delta \mathbf{x}_{0}^{(1)}:=\Delta \hat{\mathbf{x}}_{0}^{(1)}-\Delta \mathbf{x}_{0}^{(1)}=\left(\hat{\mathbf{A}}_{2}^{T} \mathbf{W}_{2} \hat{\mathbf{A}}_{2}\right)^{-1} \hat{\mathbf{A}}_{2}^{T} \mathbf{W}_{2} \boldsymbol{\varepsilon}_{2} .
$$

It is important to note that computing $E\left\{\delta \mathbf{x}_{0}^{(1)}\right\}$ in closed-form may be difficult or impossible, because both $\hat{\mathbf{A}}_{2}$ and $\boldsymbol{\varepsilon}_{2}$ contain the noise and error terms. Under the conditions that the measurement noise and error are small enough (i.e., $\Delta r_{i, j} \ll r_{i, j} \quad$ for $i=1, \ldots, M \quad$ and $j=1, \ldots, N$ and $\Delta \mathbf{x}_{0}^{(1)}(l) \ll \mathbf{x}_{0}^{(1)}(l)$ for $\left.l=1,2,3\right)$ such that we can neglect the noise and error terms in $\hat{\mathbf{A}}_{2}$ and $\hat{\mathbf{B}}_{2}, \quad \delta \mathbf{x}_{0}^{(1)}$ becomes linearly dependent on the measurement noise vector $\Delta \mathbf{r}$. In other words, when we replace $\mathbf{A}_{2}$ and $\mathbf{B}_{2}$ by $\hat{\mathbf{A}}_{2}$ and $\hat{\mathbf{B}}_{2}$, respectively, in order to simplify the error analysis, a linear relationship between $\delta \mathbf{x}_{0}^{(1)}$ and $\Delta \mathbf{r}$ is established, which is given by

$\delta \mathbf{x}_{0}^{(1)} \approx\left(\mathbf{A}_{2}^{T} \mathbf{W}_{2} \mathbf{A}_{2}\right)^{-1} \mathbf{A}_{2}^{T} \mathbf{W}_{2} \mathbf{B}_{2} \Delta \mathbf{r}$ 
Since the measurement noise vector $\Delta \mathbf{r}$ is a zero-mean random vector, $E\left\{\delta \mathbf{x}_{0}^{(1)}\right\}$ becomes zero. Therefore, by substituting (32) into (29) the error covariance matrix of the proposed estimator can be approximately expressed as follows

$$
\operatorname{cov}\left(\hat{\mathbf{x}}_{0}^{(2)}\right) \approx\left(\mathbf{A}_{2}^{T} \mathbf{W}_{2} \mathbf{A}_{2}\right)^{-1}
$$

The final point to note in this subsection is that in such a case the proposed estimator is also approximately unbiased, because

$$
\operatorname{bias}\left(\hat{\mathbf{x}}_{0}^{(2)}\right)=E\left\{\hat{\mathbf{x}}_{0}^{(2)}\right\}-\mathbf{x}_{0}=-E\left\{\delta \mathbf{x}_{0}^{(1)}\right\} \approx \mathbf{0} .
$$

\subsection{CRLB}

The CRLB establishes a theoretical lower bound on the covariance matrix of any unbiased estimator of deterministic parameters. Under the Gaussian measurement noise model, the CRLB of $\mathbf{x}_{0}$ is found by taking the inverse of the Fisher information matrix $\mathbf{J}\left(\mathbf{x}_{0}\right)$ and is given by [22]

$$
\operatorname{CRLB}\left(\mathbf{x}_{0}\right)=\mathbf{J}\left(\mathbf{x}_{0}\right)^{-1}=\left(\nabla_{\mathbf{x}_{0}}^{T}(\mathbf{r}) \mathbf{Q}^{-1} \nabla_{\mathbf{x}_{0}}(\mathbf{r})\right)^{-1},
$$

where $\nabla_{\mathbf{x}_{0}}(\mathbf{r})$ denotes the partial derivative of the true measurement vector $\mathbf{r}$ with respect to the unknown vector $\mathbf{x}_{0}$. Thus, $\nabla_{\mathbf{x}_{0}}(\mathbf{r})$ is an $M N \times 3$ matrix whose $k$ th row is given by [23]

$$
\nabla_{\mathbf{x}_{0}}(\mathbf{r})(k,:)=\boldsymbol{\rho}_{\mathbf{x}_{0}, \mathbf{x}_{t, i}}^{T}+\boldsymbol{\rho}_{\mathbf{x}_{0}, \mathbf{x}_{r, j}}^{T},
$$

where $k=(i-1) N+j$ for $i=1, \ldots, M$ and $j=1, \ldots, N$.

\subsection{Comparison with CRLB}

Now, we aim to show that the accuracy of the proposed algorithm attains the CRLB under small Gaussian noise assumption. Substituting the weighting matrix $\mathbf{W}_{2}$ given by (27) into (33) and defining $\mathbf{A}_{3}=\mathbf{B}_{2}^{-1} \mathbf{A}_{2}$ produces

$$
\operatorname{cov}\left(\hat{\mathbf{x}}_{0}^{(2)}\right) \approx\left(\mathbf{A}_{3}^{T} \mathbf{Q}^{-1} \mathbf{A}_{3}\right)^{-1} \text {. }
$$

It is worth noting that (37) and the CRLB given by (35) are of the same form. By performing some straightforward mathematical manipulations, it follows that

$$
\mathbf{A}_{3}=\nabla_{\mathbf{x}_{0}}(\mathbf{r}) \text {. }
$$

As a result, when the measurement error and noise are small, we have

$$
\operatorname{cov}\left(\hat{\mathbf{x}}_{0}^{(2)}\right) \approx \operatorname{CRLB}\left(\mathbf{x}_{0}\right) \text {. }
$$

\section{Simulations}

In this section, multiple simulation experiments are conducted to evaluate the performance of the proposed method. We compare the performance of the proposed method with that of the methods presented in [19-21] as well as the CRLB. It is shown that the proposed method outperforms the other ones. It is also indicated that there is an excellent agreement between the results of the proposed method and those of the CRLB.

Consider a distributed MIMO arrangement with $M=6$ transmitters and $N=7$ receivers. The position of all transmitters and receivers are given in Table 1 , where $R$ is the side length of each hexagon shown in Fig. 1 and is equal to $1000 \mathrm{~m}$. We have also considered two targets at positions

$$
\mathbf{x}_{0,1}=\left[\begin{array}{lll}
0.5 R & 0.5 R & 800
\end{array}\right]^{T} \mathrm{~m}
$$

and

$\mathbf{x}_{0,2}=\left[\begin{array}{lll}2 R & 2 R & 1500\end{array}\right]^{T} \mathrm{~m}$. Notice that the first target is located within the surveillance area of interest while the second one is located outside and far from the area. For better understanding, the arrangement of the transmitters, receivers, and targets in the $\mathrm{x}-\mathrm{y}$ plane is depicted in Fig. 1. The BR measurements are generated by adding zero mean Gaussian noises to the true values. The noises are considered to be dependent only on the signal-to-noise ratio (SNR) at each pair transmitter-receiver and independent of each other. Therefore, the covariance matrix of the measurements is $\mathbf{Q}=\sigma^{2} \mathbf{J}$, where $\mathbf{J}$ is an $M N \times M N$ diagonal matrix with diagonal elements equal to $\sigma_{k}^{2}=\left(\gamma r_{t, i} r_{r, j}\right)^{2}$ for $i=1, \ldots, M, j=1, \ldots, N$ and $k=(i-1) N+j$ and $\gamma$ is a constant. The localization accuracy is evaluated according to the root mean squares error (RMSE) of the target position estimate, defined as

$\operatorname{RMSE}\left(\mathbf{x}_{0}\right)=\sqrt{\sum_{l=1}^{L}\left\|\hat{\mathbf{x}}_{0,(l)}-\mathbf{x}_{0}\right\|^{2} / L}$,

where $\hat{\mathbf{x}}_{0,(l)}$ is the estimate of $\mathbf{x}_{0}$ at the $l$ th ensemble run and $L=1000$ denotes the number of ensemble runs.

This experiment is carried out to evaluate the effect of increasing the noise level in the measurements on the performance of the different algorithms. In this case, the multiplier $\sigma^{2}$ varies from $10^{-4}$ to $10^{3}$. The position RMSE of the different estimators versus $\sigma^{2}$ for both targets, $\mathbf{x}_{0,1}$ and $\mathbf{x}_{0,2}$, are represented in Fig. 2 and Fig. 3, respectively. The results show that the proposed estimator outperforms the other ones in both caseswhen the target is within or outside of the area under consideration. In both cases, we can also see that the proposed method is able to attain the CRLB accuracy in low and moderate noise conditions. As expected, the RMSE of target position estimate of the second target $\mathbf{x}_{0,2}$ is larger (about $10 \mathrm{~dB}$ ) than the RMSE of the first target $\mathbf{x}_{0,1}$. The reason is that each BR value increases for the second target and therefore for the second target at each transmitter/receiver pair the SNR decreases and the measurement noise increases.

Another simulation experiment is conducted to evaluate the performance of the proposed method for different possible positions of the target in the surveillance area. 
The target position, $\mathbf{x}_{0}=\left[x_{0}, y_{0}, z_{0}\right]^{T}$, in each ensemble run is randomly chosen from a uniform distribution such that $x_{0}, y_{0} \sim u(-2 R, 2 R) m$ and $z_{0} \sim u(0.1 R, R) m$. The empirical cumulative density function (CDF) of the different algorithms for the localization error, which is the 2-norm of the estimation error of the target position, for different noise levels (i.e., small $\sigma^{2}=10^{-2}$, moderate $\sigma^{2}=1$, and high $\sigma^{2}=10^{2}$ are shown in Figs. 4 to 6 . The results show that the proposed method has smaller position estimation error compared to the other ones for three different noise levels.

Table. 1. Position (in Meters) of Transmitters and Receivers

\begin{tabular}{cccc} 
Tx no $i$ & $x_{t, i}$ & $y_{t, i}$ & $z_{t, i}$ \\
\hline 1 & $R \cos (\pi / 6)$ & $R / 2$ & 300 \\
2 & 0 & $2 R$ & 450 \\
3 & $-R \cos (\pi / 6)$ & $R / 2$ & 600 \\
4 & $-2 R \cos (\pi / 6)$ & $-R$ & 100 \\
5 & 0 & $-R$ & 200 \\
6 & $2 R \cos (\pi / 6)$ & $-R$ & 700 \\
\hline $\mathbf{R x}$ no $j$ & $x_{r, j}$ & $y_{r, j}$ & $z_{r, j}$ \\
\hline 1 & 0 & 0 & 100 \\
2 & $2 R \cos (\pi / 6)$ & 0 & 200 \\
3 & $R \cos (\pi / 6)$ & $3 R / 2$ & 350 \\
4 & $-R \cos (\pi / 6)$ & $3 R / 2$ & 500 \\
5 & $-2 R \cos (\pi / 6)$ & 0 & 400 \\
6 & $-R \cos (\pi / 6)$ & $-3 R / 2$ & 700 \\
7 & $R \cos (\pi / 6)$ & $-3 R / 2$ & 600 \\
\hline
\end{tabular}

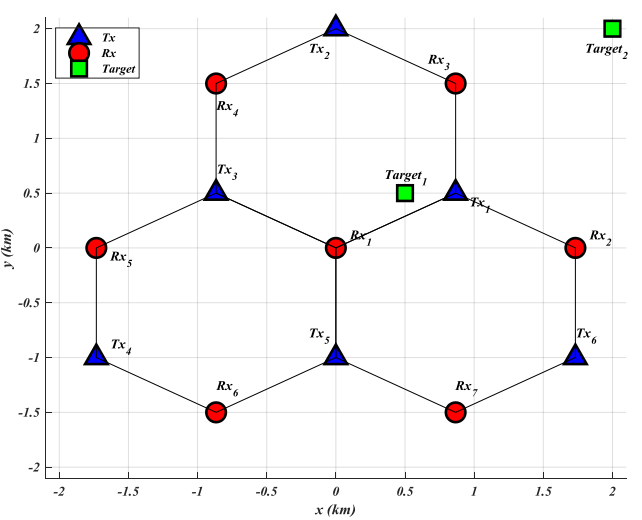

Fig. 1. Geometry of distributed MIMO radar in x-y plane

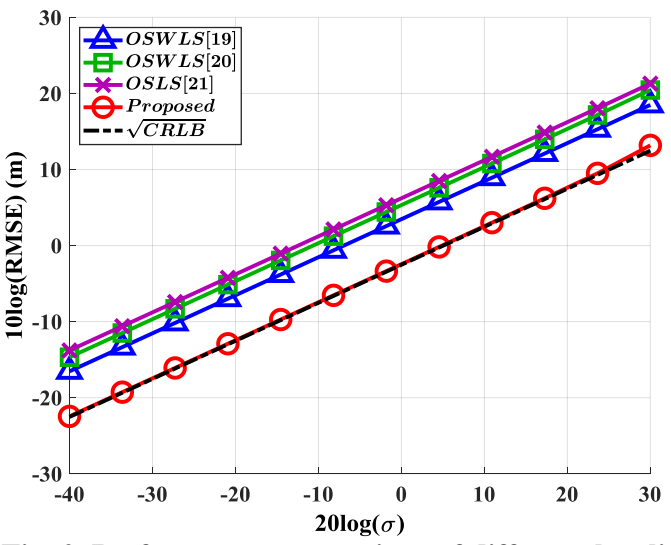

Fig. 2. Performance comparison of different localization estimators for first target $\mathbf{x}_{0,1}$

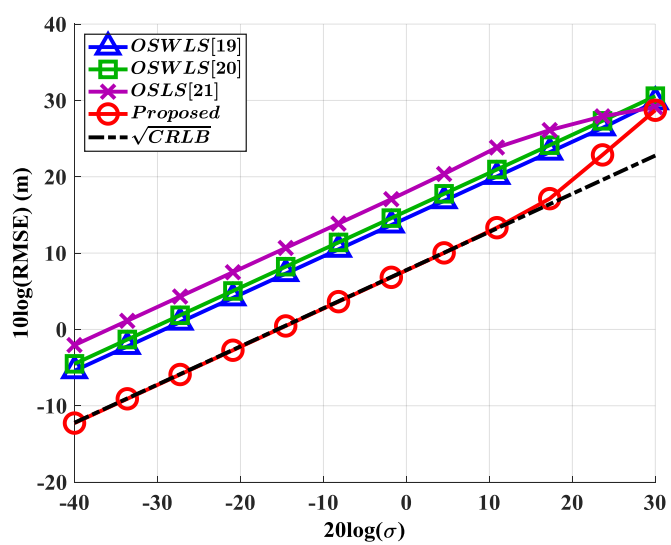

Fig. 3. Performance comparison of different localization estimators for second target $\mathbf{x}_{0,2}$

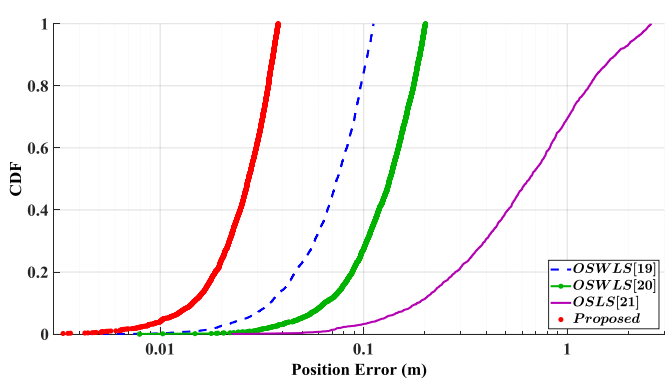

Fig. 4. Empirical CDF of position error for small noise level $\sigma^{2}=10^{-2}$

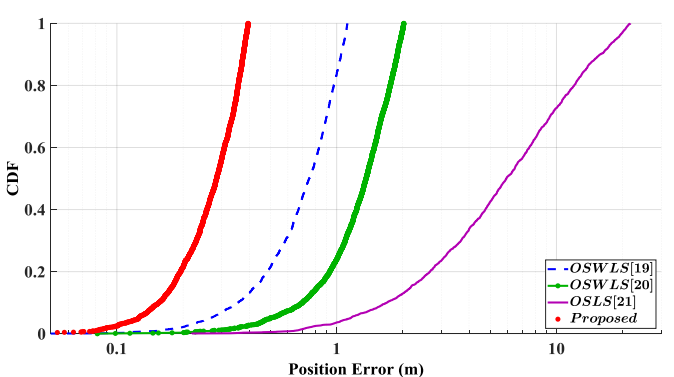

Fig. 5. Empirical CDF of position error for moderate noise level $\sigma^{2}=1$ 


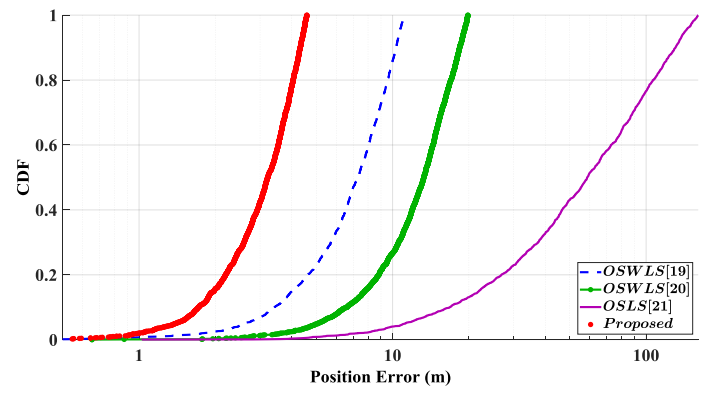

Fig. 6. Empirical CDF of position error for high noise level $\sigma^{2}=10^{2}$

\section{Conclusion}

In this paper, we developed a closed-form TSWLS solution to determine the position of a desired target in a distributed MIMO radar system using BR measurements. In the first stage, a set of linear equations from BR measurements was established by eliminating the nuisance parameters and then a WLS minimization was performed to obtain an estimate of the target position. In the second stage, by considering the relation between the nuisance parameters and the target position, we obtained an estimate the target position error and then subtracted it from the solution of the first stage to refine the target position estimate. In fact, using the second stage led to improve the localization performance of the proposed estimator such that it can attain the CRLB accuracy under low noise condition with Gaussian distribution. Numerical simulations were included to verify the theoretical developments. Simulation results represented that the proposed method outperforms the previous ones in the given examples.

\section{References}

[1] S. Pishevar, K. Mohamed-Pour, and A. Noroozi, "A closed-form two-step target localization in MIMO radar systems using BR measurements," in Electrical Engineering (ICEE), 2017 Iranian Conference on, pp. 2088-2093, May 2017.

[2] R. Amiri, F. Behnia, and H. Zamani, "Asymptotically efficient target localization from bistatic range measurements in distributed MIMO radars," IEEE Signal. Process. Lett., vol. 24, no. 3, pp. 299-303, 2017.

[3] A. Noroozi, A. H. Oveis, and M. A. Sebt, "Iterative target localization in distributed MIMO radar from bistatic range measurements," IEEE Signal. Process. Lett., vol. 24, no. 11, pp. 1709-1713, 2017.

[4] R. Amiri and F. Behnia, "An efficient weighted least squares estimator for elliptic localization in distributed MIMO radars," IEEE Signal. Process. Lett., vol. 24, no. 6, pp. 902-906, 2017.

[5] F. Liu, C. Masouros, A. Li, and T. J. I. W. C. L. Ratnarajah, "Robust MIMO beamforming for cellular and radar coexistence," IEEE Wireless Communications Letters, vol. 6, no. 3, pp. 374-377, 2017.

[6] A. Noroozi, M. A. Sebt, "Algebraic solution for threedimensional TDOA/AOA localisation in multiple-input- multiple-output passive radar," IET Radar, Sonar \& Navigation, vol. 12, no. 1, pp. 21-29, 2017.

[7] H. Yang, J. Chun, D. Chae, "Hyperbolic localization in MIMO radar systems," IEEE Antennas and Wireless Propagation Letters, vol. 14, pp. 618-621, 2015.

[8] A. Noroozi and M. A. Sebt, "Comparison between rangedifference-based and bistatic-range-based localization in multistatic passive radar," in 2015 16th International Radar Symposium (IRS), Dresden, pp. 1058-1063, Jun. 2015.

[9] H. Li, Z. Wang, J. Liu, and B. Himed, "Moving target detection in distributed MIMO radar on moving platforms," IEEE Journal of Selected Topics in Signal Processing, vol. 9, no. 8, pp. 1524-1535, 2015.

[10] E. Fishler, A. Haimovich, R. S. Blum, L. J. Cimini, D. Chizhik, and R. A. Valenzuela, "Spatial diversity in radars-models and detection performance," IEEE Transactions on Signal Processing, vol. 54, no. 3, pp. 823-838, 2006

[11] H. Godrich, A. M. Haimovich, and R. S. Blum, "Target localization accuracy gain in MIMO radar-based systems," IEEE Transactions on Information Theory, vol. 56, no. 6, pp. 2783-2803, 2010.

[12] K. Mohamedpour, and S. Fooladi Talari, "Passive Source Localization Using Joint TDOA, GROA, and AOA Measurements", Journal of Iranian Association of Electrical and Electronics Engineers, 2018.

[13] F. lotfi, A. sheikhi, and M. Biguesh, "Design of SLB with Multiple Auxiliary Antennas and Based on Detection Theory", Journal of Iranian Association of Electrical and Electronics Engineers, vol. 15, no. 2, pp. 107-115, Jul. 2018.

[14] M. Alae, H. Amindavar, "A New Method in Moving Target Recognition Using Ground Surveillance Pulse Doppler RADARs", Journal of Iranian Association of Electrical and Electronics Engineers, vol. 6, no. 2, pp. 17, Oct. 2009.

[15] A. Hokmabadi, A. Zakeri, A. Sherafat, "Sidelobe Reduction for Similar Targets in Radar Pulse Compression Using Neural Network", Journal of Iranian Association of Electrical and Electronics Engineers, vol. 14, no. 2, pp. 65-73, Sep. 2017.

[16] M. Dianat, M. R. Taban, J. Dianat, and V. Sedighi, "Target localization using least squares estimation for MIMO radars with widely separated antennas," IEEE Transactions on Aerospace and Electronic Systems, vol. 49, no. 4, pp. 2730-2741, 2013.

[17] M. Einemo and H. C. So, "Weighted least squares algorithm for target localization in distributed MIMO radar," Signal Processing, vol. 115, pp. 144-150, 2015.

[18] A. Noroozi and M. A. Sebt, "Target localization from bistatic range measurements in multi-transmitter multireceiver passive radar," IEEE Signal. Process. Lett., vol. 22, no. 12, pp. 2445-2449, 2015.

[19] A. Noroozi, M. A. Sebt, "Weighted least squares target location estimation in multi-transmitter multi-receiver passive radar using bistatic range measurements," IET Radar, Sonar \& Navigation, vol. 10, no. 6, pp. 10881097, 2016.

[20] A. Noroozi and M. A. Sebt, "Target localization in multistatic passive radar using SVD approach for eliminating the nuisance parameters," IEEE Transactions on Aerospace and Electronic Systems, vol. 53, no. 4, pp. 1660-1671, 2017.

[21] A. Noroozi and M. A. Sebt, "A new estimator for elliptic localization in distributed MIMO radar systems," in 
Electrical Engineering (ICEE), 2017 Iranian Conference on, 2017, pp. 1615-1618: IEEE.

[22] S. M. Kay, "Fundamentals of statistical signal processing, volume I: estimation theory," 1993.

[23] R. Amiri, F. Behnia and M. A. M. Sadr, "Exact Solution for Elliptic Localization in Distributed MIMO Radar Systems," in IEEE Transactions on Vehicular Technology, vol. 67, no. 2, pp. 1075-1086, Feb. 2018. 